\title{
La transdisciplinariedad y la complementariedad paradigmática, dos eslabones para la investigación científica y el desarrollo educativo universitario
}

\author{
Miguel Vásquez, PhD \\ Universidad Andina Simón Bolívar \\ miguelvasquez9@hotmail.com
}

\section{Resumen}

La transcomplejidad es una perspectiva epistemológica que busca integrar los postulados de la teoría del pensamiento complejo y de la transdiciplinariedad — que se inserta en los metadominios del conocimiento-, en la cual confluyen varias ciencias sociales. De aquí parte la construcción del conocimiento universitario entre el ser ontológico y el objeto en el mundo fenoménico, con una complementariedad paradigmática de la cual forman parte y que les permite una visión epistémica multidimensional.

Por ello, la forma de gestar investigación y conocimiento bajo esta acepción necesariamente exige repensar, en la praxis metodológica, los procesos de discusión que faciliten la enseñanza superior desde un punto de vista prospectivista, respecto de la realidad fenoménica de la universidad latinoamericana, que permitan constituir estrategias de integración colaborativa desde la ciencia, la tecnología y la sociedad (CTS).

Palabras clave: transdisciplinariedad, complementariedad, paradigma, prospectiva, ciencia, tecnología, sociedad.
Abstract
Transcomplexity is an epistemological perspective that seeks to integrate the postulates of the theory of complex thought and transdisciplinarity -which is inserted in the meta-domains of knowledge-, in which several social sciences converge. From here comes the construction of university knowledge between the ontological being and the object in the phenomenal world, with a paradigmatic complementarity of which they are part and which allows them a multidimensional epistemic vision.
Therefore, the way to conduct research and knowledge under this meaning necessarily requires rethinking, in the methodological practice, the discussion processes that facilitate higher education from a prospectivist point of view, with respect to the phenomenal reality of the Latin American university, that allow to create collaborative integration strategies from science, technology and society (CTS). 
Keywords: Transdisciplinarity, complementarity, paradigm, prospective, science, technology, society.

\section{Introducción}

La humanidad, desde el principio de sus registros epistemológicos — considerados una génesis epistemológica, una fantasía tranquilizante o una metáfora cautivadora pero, a la vez, paradójica-, desde el origen del pensamiento, nos ha encaminado a diferentes visiones filosóficas que van desde la típica dualidad —idealista-materialista, cuerpo-alma, razón-fe - hasta lo que hoy nos atañe, esa indiscutible relación entre la transcomplejidad como pieza de inicio de la transdisciplinariedad y la complementariedad paradigmática como parte de la investigación y formación educativa universitaria.

La investigación es considerada como fuente que inspira el descubrimiento y a ello agregamos la educación como método de conservación y perpetuación en la memoria histórica. La investigación científica busca conseguir conocimientos nuevos que ayuden a mejorar la calidad de vida de la sociedad mediante la universidad. Por ello, indagar y generar soluciones a problemas sociales y técnicos es parte de la evolución del conocimiento, que construye los cimientos de una nueva discusión acerca de la investigación científica, fundada en un pensamiento trascendental.

Un logro valioso es la participación sistémica que integra la interdisciplinariedad, la transdisciplinariedad y la transcomplejidad con el desarrollo multiparadigmático que permite la construcción de sistemas y modelos mediante la selección del paradigma apropiado a cada circunstancia social y educativa.

Para lograr una adecuada colaboración en la integración del conocimiento, se requiere no solo la asociación temporal e integrada de disciplinas, profesionales, escenarios y actores que nos permitan enfrentar el problema de investigación, sino también una integración permanente de concepciones filosóficas y técnicas, que generen propuestas paradigmáticas amplias, que involucren métodos, conocimientos y actitudes inmersas en una matriz cultual predominante, muy prospectivista, con ambiciones que vayan más allá de lo ya conseguido.

En nuestro mundo paralelo, los paradigmas - muy relacionados con el progreso del conocimiento científico y la educación universitaria - comparten una estrecha relación con el desarrollo de la lógica y el racionalismo, estableciendo incluso una metodología que ha predominado durante décadas, denominada "metodología científica". 
La transcomplejidad es una perspectiva epistemológica que integra los postulados de la teoría del pensamiento complejo y de la transdiciplinariedad la cual se encuentra contenida en diversos metadominios del conocimiento donde confluyen psicología, antropología, política, espiritualidad, lingüística, ecología, economía, historia, filosofía, entre otras ${ }^{1}$.

Es necesario considerar que, al emprender una investigación, la decisión de una determinada perspectiva teórico-metodológica involucra la selección de uno o varios paradigmas, cuya complementariedad hace una exclusión importante entre las miradas metodológicas y la veracidad de los datos, para aterrizar en la gestión de la educación universitaria. Enfatizando los aspectos filosóficos, tomando en cuenta la combinación de los métodos e instrumentos que correspondan a los objetivos de investigación, además permite la libertad del investigador, con un fuerte estímulo hacia la formación andragógica, basada en el ejercicio del aprendizaje sustentado en la tolerancia, la apertura práctica y la humildad mental, lo cual nos permita guiar el proceso de la investigación y de enseñanza desde una mirada transdisciplinar, con un enfoque de complementariedad paradigmática.

\section{La transdisciplinariedad en la educación universitaria: El marco de la ciencia, tecnología y sociedad}

El pensamiento complejo, según Edgar Morin, "Como si fuera un punto de un holograma, llevamos en el seno de nuestra singularidad, no solamente toda la humanidad, toda la vida, sino también casi todo el cosmos, incluyendo su misterio que yace sin duda en el fondo de la naturaleza humana. ${ }^{2}$ Este pensamiento complejo, con sus categorías de pensamiento, la transdisciplinaridad y la complementariedad paradigmática no son únicamente postulados epistemológicos o categorías del conocimiento humano, sino que son formas de ver e interpretar la realidad en la era de la posmodernidad y de la hipertecnologización de la relación e interacción, cuya mediación social es la comunicación estratégica dentro de la organización disciplinaria del saber de la complejidad de lo real.

La transdisciplinariedad asume la complejidad como forma "organizada" y en eso radica su novedad. El hombre como sistema es un ser complejo; es compleja la sociedad donde reside pero de igual forma es compleja una de sus neuronas. La complejidad como postulado científico de la transdisciplinariedad no cuestiona la ciencia clásica en su primer

1 Jean Carlos Guzmán. Metódica para abordaje una investigación desde una perspectiva transcompleja. Revista de Investigación, 2013, vol. 37, no 79, p. 29.

2 Edgar Morin. Los siete saberes necesarios para la educación del futuro. Unesco, 1999. p. 25 
principio de legislar, de ir de lo complejo a lo simple, sino de enfatizar que no son suficientes esas prácticas en el contexto científico actual. El científico contemporáneo analiza lo complejo real bajo la apariencia de lo simple. ${ }^{3}$

Esta reflexión sobre la importancia de incorporar no solo las opiniones, sino también los diferentes saberes, así como los factores internos y externos que intervienen en la correlación que origina un comportamiento determinado en una realidad particular, trae nuevas tendencias y corrientes. En ellas se analizan la interdisciplinariedad - diferentes ciencias, con un objeto de estudio diferente, cooperan entre sí- y la transdisciplinariedad - cooperación de la sociedad para entender o comprender mejor un tema complejo que requiere de diferentes tipos de saberes y su participación en condiciones de equidad- como categorías de estudio y prácticas de las ciencias actuales.

La interdisciplinariedad implica puntos de contacto entre las disciplinas en los que cada una aporta sus problemas, conceptos y métodos de investigación. La transdisciplinariedad, sin embargo, es lo que simultáneamente le es inherente a las disciplinas y donde se termina por adoptar el mismo método de investigación. La transdisciplinariedad está entre las disciplinas, en las disciplinas y más allá de las disciplinas. Las definiciones son mucho más complejas y nos obligan a repasar históricamente su aparición y uso. ${ }^{4}$

La complejidad está presente en todas partes de la sociedad. Cuando se inicia el análisis a partir de la física cuántica, así como en las reflexiones sociales, lo complejo se da en el mundo macrofísico y microfísico. Uno de los mayores pensadores de la complejidad es Edgar Morin, quien ve al mundo como un todo indisociable, donde nuestro ser individual posee conocimientos ambiguos, desordenados, que necesita acciones de retroalimentación y propone abordajes de formas multidisciplinaria y multirreferenciada, para construir el pensamiento desarrollado con un profundo análisis lleno de elementos de certeza epistémica.

Es valioso discutir sobre la base del pensamiento complejo y la trandisciplinariedad, un currículo integrador sociocrítico y liberador. No está muy distante pensar en un currículo por complejidades, el cual conduzca a pensar que un problema educativo deberá tener una o varias soluciones complejas basadas en la investigación científica.

3 Nuria Esther Pérez Matos y Emilio Setién Quesada. "La interdisciplinariedad y la transdisciplinariedad en las ciencias: una mirada a la teoría bibliológico-informativa". Acimed 18 n. 4 (2008) p. 10.

4 Ibid. p.2 
El último grado de coordinación susceptible de existir en el sistema de educación/innovación, coordinación que pudiéramos llamar transdisciplinariedad, no dependería solamente de una axiomática común derivada de una coordinación hacia un "propósito global del sistema", sino también del reforzamiento mutuo de epistemologías en ciertas áreas... En el conjunto del sistema, los conceptos y principios de la transdisciplinariedad cambian significativamente cuando hay cambios en el "objetivo global del sistema" hacia el cual se dirige la función de coordinación de la "significación" que está en la cima del sistema. ${ }^{5}$

Es importante considerar que es necesario promover la generación y utilización de la transdisciplinariedad en un ámbito más amplio, como un metaparadigma para conjugar la interrelación entre el enfoque, la metodología y su participación en el sistema, con un nuevo y actual modo de pensar sistémico.

Para lograr que la participación sistémica, integrando la interdisciplina, la transdisciplina y la transpersonalidad, colaboren eficiente y eficazmente a la integración del conocimiento, requiere no solo de la asociación conjunta temporal de disciplinas, profesiones, religiones, sectas y actores, para enfrentar un problema, sino requiere promover una integración permanente evolutiva, que produzca una síntesis paradigmática amplia de métodos, conocimientos y entendimientos, más allá de lo ya alcanzado. ${ }^{6}$

Un pensamiento sistémico es importante cuando se refiere a los modelos educativos, los cuales deben estar orientados hacia la complejidad. Se ha evidenciado la escasa orientación a escala mundial de este enfoque, que permite la ruptura de esquemas, ideologías y construcciones reduccionistas de la ciencia. Lo ideal es conseguir que los estudiantes desarrollen su sensibilidad social cognitiva bajo el enfoque de la incertidumbre, desarrollen su diálogo interno y externo tomando en consideración su ser complejo.

Solo con la inter y la transdisciplinariedad puede "reavivarse" el sistema de educación e innovación, en el sentido de que los contenidos, estructuras y puntos de unión de las disciplinas, cambian continuamente a través de una coordinación dirigida hacia la consecución de un propósito común del sistema. Así pues, la inter y la transdisciplinariedad

$5 \quad$ Erich Juntsch, "Hacia la interdisciplinariedad y la transdisciplinariedad en la enseñanza y la innovación”, en Interdisciplinariedad: problemas de la enseñanza y la investigación en las Universidades, edit. Léo Apostel, Guy Berger, Asa Briggs y Guy Michaud, 110-41 (México: Asociación Nacional de Universidades e Institutos de Enseñanza Superior, 1979). p. 9

6 Ricardo A. Estrada García y Germán S. Monroy Alvarado, "Perspectivas múltiples, multi-paradigmas y participación sistémica en las organizaciones" (ponencia en VI Congreso Nacional y I Internacional de Investigación en Ciencias Administrativas, Paradigmas emergentes de la administración en las sociedades del conocimiento, CDMX, 24-26 de abril de 2002). p.11 
se convierten en las nociones claves para intentar un acercamiento de análisis de sistemas, a la educación y la innovación. ${ }^{7}$

Por otra parte, esto quiere decir que se requiere superar dos de los mayores obstáculos que surgen en el camino hacia la inter y la transdisciplinariedad: la rigidez de las disciplinas, los conceptos y la axiomática disciplinaria, desarrollados en los niveles inferiores; y el otro es la aplicación de los conceptos y la axiomática de los niveles inferiores, a los niveles superiores. $^{8}$

Lo expuesto se complementa con lo que Edgar Morin señala respecto de la sociedad, enfocándola más allá de las concepciones tradicionalistas, como

Una sociedad planetaria cuyas diferencias internas quedan supeditadas a los atisbos - ya un tanto silentes - de las características propias geopolíticas y culturales que penetran sus raíces en los más profundo de su tierra, pero que se yerguen en el horizonte de un mundo infinito en posibilidades. Una sociedad local y a la vez universal que avanza sin cansancio hacia la denominada unitas multiplex, es decir, hacia la unidad sustentada en la diversidad humana. ${ }^{9}$

Se vislumbra la necesidad de construir una epistemología de la creatividad educativa, donde la transdisciplinariedad y el enfoque de complejidad, con la complementariedad paradigmática en la educación universitaria, se conviertan en poderosos referentes metodológicos y andragógicos para las múltiples aplicaciones en la enseñanza superior de la ciencias con un eje paradigmático de la incorporación de las emociones, por medio de la neurociencia y la conciencia, con aplicación de la ética como intangibles.

Según Jessica Cabrera Cuevas y Agustín de La Herrán Gascón, "tanto la transdisciplinariedad como el enfoque de complejidad evolutiva son potentes referentes epistemológicos, metodológicos y prácticos para las múltiples aplicaciones de la creatividad, en especial, en la aplicación social y de contextos de innovación didáctica"; ${ }^{10}$ por ello, es indispensable que se promueva la evaluación y control social del desarrollo tecnoeducativo y científico.

7 Erich Juntsch, "Hacia la interdisciplinariedad y la transdisciplinariedad en la enseñanza y la innovación”, en Interdisciplinariedad: problemas de la enseñanza y la investigación en las Universidades, edit. Léo Apostel, Guy Berger, Asa Briggs y Guy Michaud, 110-41 (México: Asociación Nacional de Universidades e Institutos de Enseñanza Superior, 1979). p. 10

8 Ibid. p. 10

9 Gil, O. R. La sociedad transcompleja y la praxis andragógica en la Educación Superior. Fermentum, 2005, no 43, p. 5

10 Jessica Cabrera Cuevas y Agustín de La Herrán Gascón, “Creatividad, complejidad y formación: un enfoque transdisciplinar”, Revista Complutense de Educación 26 n. 3 (2015) p. 505 
La transcomplejidad aplicada a los estudios de la ciencia, tecnología y sociedad (CTS) es un campo considerable del ejercicio académico y profesional en la investigación académica, política, profesional, de la educación, entre otros de una ilimitada heterogeneidad, en la teoría, metodología y la ideología que intenta explicar los puntos importantes del fenómeno científico y tecnológico. Por su parte, Crisálida Villegas ${ }^{11}$ considera que puede ser asumido en un enfoque transcomplejo y transdisciplinario.

Por lo expuesto, la educación universitaria latinoamericana es una gran cantera para desarrollar la ciencia y la tecnología de acuerdo con nuestra sociedad. No debemos buscar adaptar nuestra sociedad a la ciencia y tecnología foránea, sino construir enfoques de complementariedad paradigmática que nos permita reconstruir los currículos y las metodologías de enseñanza andragógicas de la ciencia y la tecnología, en módulos y materias inter y transdisciplinarios.

No puede pretenderse una renovación crítica de la docencia restringiendo tal cambio solo a los contenidos [...] involucra entonces el abandono del papel del profesor como metaexperto [...] por un lado, y el estímulo de la participación crítica y creativa de los estudiantes en la organización y desarrollo de la docencia, por otro. La actitud crítica y participativa debería entonces ser reflexiva y alcanzar a la propia metodología docente y a las técnicas didácticas. ${ }^{12}$

Es el sueño de todo docente comprometido con la mística de la enseñanza, generar un cambio positivo de la sociedad mediante la educación universitaria, con una actualización epistemológica, axiológica, andragógica y didáctica que le permita desarrollar la ciencia y tecnología socialmente comprometidas con nuestra realidad latinoamericana.

Es necesario continuar investigando con enfoque transdisciplinar e integrar la complementariedad paradigmática hacia una complejidad evolutiva en lo que respecta a programas de educación y formación en general, que permitan promover cambios positivos hacia la sociedad, en un contexto de libertad, ética y valores humanos, conjugados con la generosidad académica de los investigadores y marcado por un permanente intercambio de conocimiento compartido.

11 Crisálida Villegas, "La educación y los estudios de ciencia, tecnologia, sociedad (CTS) en el marco de la transcomplejidad", Redtive, 12 de mayo de 2014. https://reditve.wordpress.com/2014/05/12/laeducacion-y-los-estudios-de-ciencia-tecnologia-sociedad-cts-en-el-marco-de-la-transcomplejidad/

12 López Cerezo, José A., José A. Méndez Sanz y Oliver Todt, "Participación pública en política tecnológica: problemas y perspectivas”, Arbor 159 n. ${ }^{\circ} 627$ (1998) p.7. 
Con este enfoque paradigmático de la transdisciplinariedad y la complementariedad paradigmática, la epistemología aparece con una alta posibilidad de formar, educar e investigar sobre la base de las emociones y la conciencia, donde la ética, los valores y las virtudes del ser ontológico participen en forma activa en el desarrollo profesional de los docentes reconociendo, valorando el potencial cognitivo y creativo de los estudiantes y les permita compartir su conocimiento, para el beneficio de la ciencia, la sociedad y la tecnología, cercanos y mediatos de los cuales son parte.

De acuerdo con Crisálida Villegas, una educación que considera la integración de criterios en relación con las temáticas CTS, naturaleza y cultura, cuya distinción epistemológica entre ciencias naturales y sociales, tecnologías materiales y sociales, así como entorno y persona, permite incluir la complementariedad paradigmática, en su proceso y metodología cognitiva.

En América Latina, atravesamos un contexto educativo complejo, con una emergencia de saberes integrados e intervinculados recíprocamente. Se requieren procesos inter, multi y transdisciplinarios, con un enfoque de complementariedad paradigmática, en que la ciencia, la tecnología y la sociedad y sus disciplinas asociadas se entretejen en conjunto. Las interrelaciones que se generan entre las problemáticas sociales, ambientales y humanas en Latinoamérica pueden plantear una nueva configuración. Ya lo propuso Barbero con las mediaciones sociales; un interfaz que permita relacionar el conocimiento de cada disciplina con el conocimiento de todas y viceversa, mediante un proceso de complementariedad paradigmática que, a la vez, se complementan y se excluyen.

\section{La complementariedad paradigmática y su aporte para la investigación científica y la educación universitaria}

La humanidad, desde el principio de sus registros epistemológicos, ha considerado la formación como una fantasía tranquilizante de supervivencia, una génesis epistemológica o una metáfora cautivadora pero a la vez paradójica. Si nos detenemos a pensar en la investigación, nos lleva a diferentes visiones filosóficas que van desde la típica dualidad idealista-materialista, cuerpo-alma, razón-fe- que todas comparten en su mismo principio occidental. Pero lo que nos atañe es la

Indiscutible relación entre el desarrollo epistemológico, la consolidación de territorios y la estructuración política dentro de ellos; necesita reconocer la endeble línea entre el poder y la construcción histórica del pensamiento y más aún cuando las nociones 
quizá más tradicionales y prioritarias en un recorrido de este calibre, las concentran las definiciones de investigación y educación. ${ }^{13}$

La investigación es considerada como fuente que inspira el descubrimiento y a ello agregamos la educación como método de perduración, conservación y perpetuación en la memoria histórica de la sociedad. Es decir, la investigación científica busca conseguir conocimientos nuevos que ayuden a mejorar la calidad de vida de la sociedad. Por ello, indagar y generar soluciones a problemas sociales y técnicos es parte de la evolución del conocimiento, construyendo los cimientos de una nueva discusión acerca de la investigación científica, fundada en un pensamiento trascendental.

Actualmente se trabajan distintos conceptos sobre la base del proceso investigativo, en lo referido a la epistemología y al protocolo de la investigación educativa, como quehacer científico en el campo de la educación, cuyo proceso de reflexión es sumado a la colaboración e integración del pensamiento y criterios de todos los actores del hecho educativo que se investiga y que proporciona elementos de mayor atención a los procesos de construcción del conocimiento, que hoy se caracterizan por una creciente complejidad.

Al interior de la investigación, el accionar de la educación, desde la visión de la complejidad, es la episteme educativa perseguida. Si tomamos en cuenta el proceso histórico de aplicación del currículo en la educación, podemos evidenciar, en un inicio, el aparecimiento de un currículo basado en propósitos; posteriormente surgió otro basado en objetivos de aprendizaje; a continuación toma posición el currículo basado en competencias; y actualmente está de moda el currículo basado en capacidades. Los dos primeros buscan el cumplimiento de metas en lo cognitivo; y los dos currículos finales se enfocan en las habilidades y destrezas de los que aprenden.

Esto se complementa con lo que Edgar Morin señala respecto de la sociedad, enfocándola más allá de las concepciones tradicionalistas, como:

Provocar un cambio de paradigma que se oriente hacia el pensamiento complejo de una sociedad tecnológica, a otra transcompleja, es tarea de la academia contemporánea y de sus integrantes. Pensar en una educación para una sociedad planetaria que propicie los caminos para alcanzar "una mundología de la vida cotidiana" ${ }^{14}$ no puede sostenerse en función de un desarrollo que excluya al ser humano, porque la brecha existente entre el ser

13 Juan Manuel Martínez Herrera, "Investigación educativa, panorama histórico y contexto", Mi Ratón 10 (2010), https://www.utp.edu.co/educacion/raton/antes/miraton10/textos/investigacion\%20educativa.pdf, p.1

14 Edgar. Morin. Educar en la era planetaria. Editorial Gedisa, 2003.p.123 
y su naturaleza social se acentuaría, desconociendo la interacción plena entre los diversos elementos que intervienen en la sociedad compleja y transdisciplinar.

Esto, como preámbulo, nos permite corroborar lo expresado por Ernesto E. Hashimoto y Sarita Saavedra Grández:

La complementariedad no es solo autodesarrollo, sino implica el desarrollo de los otros y con los otros, y desde esa perspectiva la complementariedad es positiva. En cierta medida, es una perspectiva "natural" con la que la persona debe visionar y estudiar una realidad. ${ }^{15}$

En una primera aproximación, es muy común concebir como pensamiento dominante que los paradigmas de investigación son excluyentes. Se concibe a los paradigmas como si fuesen líneas paralelas que jamás se entrecruzarán y, para completar el panorama, se piensa que se repelen. Se los mira como antagónicos e irreconciliables, un discurso trabajado desde las emociones y no desde la visión de complementariedad paradigmática. Se asume que el conocimiento que cada paradigma genera la verdad y lo único valioso, y el del resto de conocimiento que pueden generar otras corrientes o paradigmas es solamente una falacia o un conocimiento degradado, sin rigor científico.

Con el devenir de los procesos de investigación, surge la necesidad de pensar en una nueva forma de investigar, donde una sola forma de investigar es insuficiente para entender los problemas, por lo que se utiliza la investigación cuanti-cualitativa o multimetodológica, que aparece como una corriente que promueve la integración mediante la combinación de paradigmas.

Esta corriente de pensamiento, según Ernesto E. Hashimoto y Sarita Saavedra Grández, forma parte de la propuesta de la complementariedad en la investigación y busca resolver el problema de la exclusión, el desorden y la confusión. ${ }^{16}$

15 Ernesto E. Hashimoto y Sarita Saavedra Grández, "La complementariedad paradigmática: Un nuevo enfoque para investigar", en Memorias de ponencias II Congreso de la Red Ecuatoriana de Universidades y Escuelas Politécnicas para Investigación y Postgrados, IV Congreso Binacional de Ciencia, Tecnología e Innovaciones de las Universidades del Sur del Ecuador y del Norte del Perú, edit. Coordinación del congreso REDU, 277-8 (Loja: UTPL, 2014). p. 13 


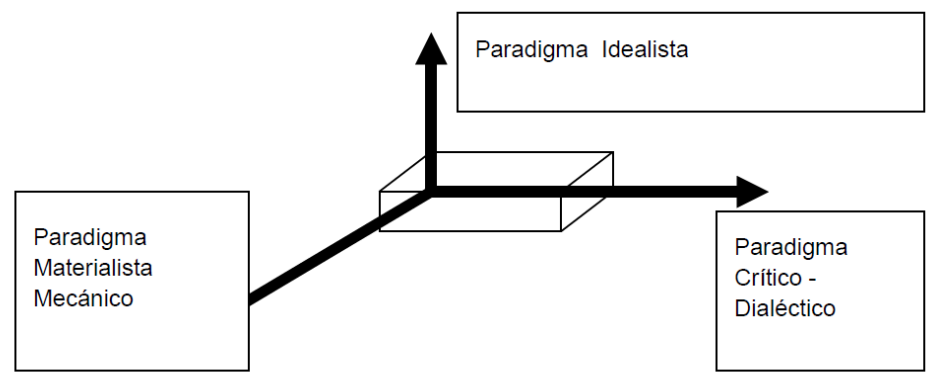

Fuente: Hashimoto y Saavedra Grández, "La complementariedad paradigmática”.

Lo que Ernesto E. Hashimoto y Sarita, Saavedra Grández proponen es que, antes de promover el "choque o conflicto paradigmático" o la mezcla paradigmática sin sentido, se propone la complementariedad o "alianza paradigmática" entre dos o tres paradigmas en los procesos de investigación. ${ }^{17}$ De esta manera, el investigador puede ejercer su actividad, tomando en cuenta su posición paradigmática y permitiendo el acompañamiento de otros paradigmas para contar con un conocimiento integral y complementario del objeto de estudio.

Expuesto de esta manera, nos refiere que, cuando la selección de una metodología no se la determina exclusivamente por la fidelidad a un paradigma, en el cual el investigador no piensa que debería incorporar un paradigma extremo, con exclusión del otro, entonces no hay ningún motivo para que el investigador cuente con algún impedimento para adherirse a cualquier enfoque paradigmático, a una postura ontológica y a los procesos de una metodología cualitativa, en oposición a otra cuantitativa, o viceversa.

En vista de la complejidad que representan los procesos sociales y de la diversidad en la experiencia científica, unida a que ningún método es la única vía de conocimiento, la complementariedad paradigmática se muestra como un estilo de este nuevo modo cognitivo que incorpora el desarrollo de estrategias investigativas múltiples para producir y validar el conocimiento, con formas distintas de acercarse a la realidad pero complementarias, los enfoques cuantitativo y cualitativo.

La producción de conocimiento desde la visión de la complementariedad metodológica es posible a través de la práctica multimétodo, un ejercicio investigativo que alude al uso de diversos métodos y procedimientos conocido también en la literatura especializada como enfoque múltiple, diseño mixto, integrado o multimodal. En torno a los elementos que intervienen en la toma de decisiones para la aplicación de la estrategia 
multimétodo resaltan principalmente tres; la dimensión metodológica, las estrategias básicas de integración y la fase en la cual se ejecuta; en la práctica, esto se produce cuando el investigador integra diferentes estrategias procedentes de un mismo enfoque de investigación o de enfoques distintos. En el primer caso, la orientación del multimétodo es intraparadigmática e interparadigmática en el segundo. ${ }^{18}$

Frente a este nuevo proceso recursivo, donde los paradigmas visibilizan los problemas sociales, cuyas nuevas realidades sociales exigen nuevos paradigmas, con la incorporación de herramientas y TIC en lo referente a la educación, donde recogen esta complejidad, que posibilitan y fortalecen el "diálogo" con los "otros" y con nosotros mismos como inestimable instrumento de reflexión. Complementando con una revisión de los principales

sistemas de evaluación que anteponen la rigurosidad metodológica (metodolatría) reconociendo la multiplicidad paradigmática e incorporando el diálogo como instrumento de descubrimiento, de "toma de conciencia" y de "conversión", con fuertes y cada vez más profundos contactos entre lo que hasta ahora se ha distinguido como cultura de especialistas y cultura general. ${ }^{19}$

Para corroborar, se indica que, en la misma, se ubican, bajo la consideración de una relativa complementariedad paradigmática, los marcos clásicos del conocimiento científico social de raigambre positivista, el marco del materialismo y la teoría crítica y el reflexivo interpretativo bajo una convivencia propia de los períodos de ciencia normal para el caso de las ciencias sociales. ${ }^{20}$

En materia de educación, se concibe el aprendizaje como una transformación, una modificación celular que no es posible de producir espontáneamente por un individuo solitario, por más que se piense en alguna capacidad resiliente como efecto de la exclusiva inclinación personal. Se piensa que ahora, ya nadie quiere educar a nadie, así como nadie se educa por su cuenta, los seres humanos se educan en conjunto, compartiendo saberes, inmersos en la sociedad que es el medio.

La base de la innovación educativa es la investigación, con su inclinación a pensar que el cambio social y el mejoramiento educativo son factibles y más aún si vemos que su paradigma es una base corpórea y material. Además, su estilo interdisciplinar y de

18 Neligia Blanco y Johann Pirela, "La complementariedad metodológica: Estrategia de integración de enfoques en la investigación social", Espacios Públicos 19 n. ${ }^{\circ} 45$ (2016). P. 97

19 María de los Ángeles Sagastizabal y Claudia Liliana Perlo, "Contribuciones para la construcción de una metodología de la complejidad" (ponencia XIII Jornadas de Investigación y II Encuentro de Investigadores en Psicología del Mercosur, Buenos Aires, 9 de enero de 2006). p.6.

20 Luis Hernán Santarsiero, “Aportes para una revisita a los métodos cualitativos y sus trayectorias: Reseña sobre Estrategias de investigación cualitativa, coord. Irene Vasilachis", Revista Latinoamericana de Metodología de las Ciencias Sociales 1 n.o 1 (2011): 120-125. http:// www.fuentesmemoria.fahce.unlp.edu.ar/art_revistas/pr.4831/pr.4831.pdf. 
complementariedad paradigmática se convierte en adecuado espacio para sostener la innovación y los ansiados cambios paradigmáticos en la educación. ${ }^{21}$

Los problemas que presenta la investigación en educación generalmente son políticos, económicos, de formación, culturales y de la gestión misma de las investigaciones; por ello, es valioso abordar la educación universitaria desde la complementariedad paradigmática en la investigación, como una práctica de libertad, en la que la comunicación, como eje articulador, es vital para entablar el diálogo, que debe ser

el motor de la reflexión crítica de la realidad de la socidad, donde los sujetos tengan un mismo bien común, basado en valores éticos. Es aquí en donde reside una diferencia fundamental con el conocimiento y aprehensión de las Ciencias Naturales y Exactas, las cuales no admiten interpretación más allá de las leyes y principios que rigen los fenómenos y procesos. ${ }^{22}$

Es urgente la necesidad de que la investigación utilice la complementariedad paradigmática para generar procesos de investigación transdisciplinarios en educación, donde la docencia universitaria debe mejorar y estar abierta a nuevas transformaciones, por el impacto que generan las nuevas tecnologías, uso de la andragogía en la formación, desarrollo de competencias y el profesionalismo entre los futuros egresados exigen la renovación y reinvención de los métodos y formaciones educativas.

\section{Conclusiones}

La transdisciplinariedad es una práctica que configura formas de investigación y aprendizajes, que debe estar presente en el proceso de enseñanza-aprendizaje universitario, donde se expresa la integralidad entre el método y el contenido, lo que permite que se actúe de forma consciente o inconsciente entre el sujeto que aprende y el que enseña, por medio de contenidos disciplinares basados en el pensamiento complejo. Por ello, se afirma que la transdisciplinariedad no busca mantener el dominio de varias disciplinas inmersas en el proceso; sino abrir todas las disciplinas a sus puntos en común para, juntas, ir más allá de sus fronteras.

La investigación en la academia, inmersa en una sociedad transcompleja e interrelacionada, debe preocuparse por generar profesionales conscientes de su relación con el caos y la incertidumbre actual. Ciudadanos capaces de comunicar al ser ontológico su

21 Venegas Traverso, Cristián Alejandro. "La complementariedad científica de Francisco Varela y Paulo Freire para las prácticas educativas innovadoras". Theoria 21 n. ${ }^{\circ} 1$ (2012): 37-50.

22 Gil Flores, Javier. “La evaluación de competencias laborales”. Educación XX1 10 (2007): 83-106. 
rol de mejorar y gestionar el buen vivir en el marco de la tecnociencia y el impacto en el uso y abuso de recursos naturales, pensando en las futuras generaciones.

Los estudios de ciencia, tecnología y sociedad son un campo especial de trabajo en investigación de una extraordinaria heterogeneidad teórica, metódica e ideológica, que trata de interpretar los espacios de los fenómenos científicos y tecnológicos. Según Jorge, Núñez, su "enfoque general es de carácter critico [...] e interdisciplinar"23. Por su parte, Crisálida, Villegas considera que puede ser asumido en un enfoque transcomplejo y transdisciplinario, como un interfaz que permita relacionar el conocimiento de cada disciplina con el conocimiento de todas y viceversa, mediante un proceso de complementariedad paradigmática que permite se complementen y excluyan, que lo incorpora a esta discusión.

Es importante destacar que se debe considerar una mayor participación de los actores involucrados en el proceso educativo universitario, incluyendo a todo su entorno social y físico, su cultura y creencias, como parte del proceso de una investigación colaborativa, donde la complementariedad paradigmática, orientada a procesos de diálogo y reflexión profunda, genere formas de pensamiento nuevas respecto de la enseñanza universitaria, con uso de la andragogía, que involucre prácticas innovadoras, que generen compromiso y sensibilidad social de los futuros profesionales con los sujetos sociales, que deriven en el establecimiento de una sociedad incluyente e integradora.

23 Núñez, Jorge. La ciencia y la tecnología como procesos sociales: Lo que la educación científica no debe olvidar. Ciudad: Organización de Estados Iberoamericanos, 2011. p. 5 


\section{Bibliografía}

Blanco, Neligia y Pirela, Johann. "La complementariedad metodológica: Estrategia de integración de enfoques en la investigación social”, Espacios Públicos 19 n. ${ }^{\circ} 45$ (2016).

Cabrera Cuevas Jessica y De La Herrán Gascón, Agustín, “Creatividad, complejidad y formación: un enfoque transdisciplinar", Revista Complutense de Educación 26 n. ${ }^{\circ} 3$ (2015)

Estrada García, Ricardo A. y Monroy Alvarado, Germán S. "Perspectivas múltiples, multiparadigmas y participación sistémica en las organizaciones" (ponencia en VI Congreso Nacional y I Internacional de Investigación en Ciencias Administrativas, Paradigmas emergentes de la administración en las sociedades del conocimiento, CDMX, 24-26 de abril de 2002).

Gil Flores, Javier. "La evaluación de competencias laborales". Educación XX1 10 (2007): 83-106.

Gil, O. R. La sociedad transcompleja y la praxis andragógica en la Educación Superior. Fermentum, 2005, no 43.

Guzmán, Jean Carlos. Metódica para abordaje una investigación desde una perspectiva transcompleja. Revista de Investigación, 2013, vol. 37, no 79.

Hashimoto, Ernesto E. y Saavedra Grández, Sarita, "La complementariedad paradigmática: Un nuevo enfoque para investigar", en Memorias de ponencias II Congreso de la Red Ecuatoriana de Universidades y Escuelas Politécnicas para Investigación y Postgrados, IV Congreso Binacional de Ciencia, Tecnología e Innovaciones de las Universidades del Sur del Ecuador y del Norte del Perú, edit. Coordinación del congreso REDU, 277-8 (Loja: UTPL, 2014).

Juntsch, Erich "Hacia la interdisciplinariedad y la transdisciplinariedad en la enseñanza y la innovación", en Interdisciplinariedad: problemas de la enseñanza y la investigación en las Universidades, edit. Léo Apostel, Guy Berger, Asa Briggs y Guy Michaud, 11041 (México: Asociación Nacional de Universidades e Institutos de Enseñanza Superior, 1979).

López Cerezo, José A. Méndez Sanz y Oliver Todt, "Participación pública en política tecnológica: problemas y perspectivas", Arbor 159 n. ${ }^{\circ} 627$ (1998)

Martínez Herrera, Juan Manuel. "Investigación educativa, panorama histórico y contexto", Mi Ratón $10 \quad$ (2010), https://www.utp.edu.co/educacion/raton/antes/miraton10/textos/investigacion\%20ed ucativa.pdf

Morin, Edgar. Educar en la era planetaria. Editorial Gedisa, 2003.

Morin, Edgar. Los siete saberes necesarios para la educación del futuro. Unesco, 1999.

Núñez, Jorge. La ciencia y la tecnología como procesos sociales: Lo que la educación científica no debe olvidar. Ciudad: Organización de Estados Iberoamericanos, 2011.

Pérez Matos, Nuria Esther y Setién Quesada, Emilio. "La interdisciplinariedad y la transdisciplinariedad en las ciencias: una mirada a la teoría bibliológico-informativa". Acimed 18 n. ${ }^{\circ} 4$ (2008).

Sagastizabal, María de los Ángeles y Perlo, Claudia Liliana. "Contribuciones para la construcción de una metodología de la complejidad" (ponencia XIII Jornadas de Investigación y II Encuentro de Investigadores en Psicología del Mercosur, Buenos Aires, 9 de enero de 2006).

Santarsiero, Luis Hernán. "Aportes para una revisita a los métodos cualitativos y sus trayectorias: Reseña sobre Estrategias de investigación cualitativa, coord. Irene Vasilachis", Revista Latinoamericana de Metodología de las Ciencias Sociales 1 n.o 
www.fuentesmemoria.fahce.unlp.edu.ar/art_revistas/pr.4831/ pr.4831.pdf.

Venegas Traverso, Cristián Alejandro. "La complementariedad científica de Francisco Varela y Paulo Freire para las prácticas educativas innovadoras". Theoria $21 \mathrm{n} .^{\circ} 1$ (2012): 37-50.

Villegas, Crisálida. "La educación y los estudios de ciencia, tecnología, sociedad (CTS) en el marco de la transcomplejidad", Redtive, 12 de mayo de 2014. https://reditve.wordpress.com/2014/05/12/la-educacion-y-los-estudios-de-cienciatecnologia-sociedad-cts-en-el-marco-de-la-transcomplejidad/ 